\title{
Optical absorption in Fibonacci lattices at finite temperature
}

\author{
A. Rodríguez \\ Departamento de Matemática Aplicada y Estadística and Grupo Interdisciplinar de Sistemas Complicados, Universidad Politécnica, \\ E-28040 Madrid, Spain \\ F. Domínguez-Adame \\ Departamento de Física de Materiales and Grupo Interdisciplinar de Sistemas Complicados, Universidad Complutense, \\ E-28040 Madrid, Spain \\ (Received 12 June 1997)
}

\begin{abstract}
We consider the dynamics of Frenkel excitons on quasiperiodic lattices, focusing our attention on the Fibonacci case as a typical example. We evaluate the absorption spectrum by solving numerically the equation of motion of the Frenkel-exciton problem on the lattice. Besides the main absorption line, satellite lines appear in the high-energy side of the spectra, which we have related to the underlying quasiperiodic order. The influence of lattice vibrations on the absorption line shape is also considered. We find that the characteristic features of the absorption spectra should be observable even at room temperature. Consequently, we propose that excitons act as a probe of the topology of the lattice even when thermal vibrations reduce their quantum coherence. [S0163-1829(97)07238-X]
\end{abstract}

\section{INTRODUCTION}

Low-dimensional quasiperiodic systems have been the subject of intensive theoretical studies because of their unusual properties, such as fractal energy spectra and selfsimilar wave functions. Several electronic properties of these systems can be inferred from optical measurements: In particular, optical-absorption techniques are suitable for their characterization because quasiperiodic order causes the occurrence of well-defined lines which do not arise in periodic or random systems. ${ }^{1}$ In previous works ${ }^{1-4}$ we have focused our attention on Frenkel excitons in Fibonacci lattices as typical examples of low-dimensional quasiperiodic systems. By solving numerically the equation of motion of Frenkel excitons on the lattice, in which on-site energies take on two values following the Fibonacci sequence, we found that the characteristic satellites observed in the high-energy side of the absorption spectra correspond to well-defined peaks of the Fourier transform of the lattice. This result is important since it enables us to find a simple relationship between the underlying long-range order and optical measurements. Due to the long-range nature of Fibonacci order, it is clear that quantum coherence of excitons over large distances is required to observe these characteristic features. In most systems, however, there are inelastic-scattering mechanisms which could result in a reduction of the coherence length. Among them, the effect of lattice vibrations on the opticalabsorption line shape has long been a subject of special interest. $^{5}$

In this paper we investigate optical absorption due to Frenkel excitons in Fibonacci lattices at finite temperature. To this end, we consider the Frenkel Hamiltonian and include the exciton-phonon interaction. We follow closely the approach previously introduced by Schreiber and Toyozawa ${ }^{5}$ in periodic lattices to take into account exciton-phonon interaction. We use a general treatment to study the dynamics of Frenkel excitons in these lattices, solve the microscopic equation of motion proposed by Huber and Ching, ${ }^{6}$ and find the optical-absorption spectrum. The optical line shape at different temperatures is obtained. The main aim of the paper is to demonstrate that the satellite peaks appearing in the high-energy portion of the spectrum, ${ }^{1}$ which characterize the long-range order of the lattice, are still present and observable at finite temperature. If so, we will have shown that excitons act as a probe of the lattice structure even at finite temperature.

\section{PHYSICAL MODEL AT ZERO TEMPERATURE}

We consider a system of $N$ optically active centers occupying positions on a linear regular lattice. The Frenkel Hamiltonian describing this system can be written as follows (we use units such that $\hbar=1$ )

$$
\mathcal{H}=\sum_{n} V_{n} a_{n}^{\dagger} a_{n}-J \sum_{n}\left(a_{n}^{\dagger} a_{n+1}+a_{n+1}^{\dagger} a_{n}\right)
$$

Here $a_{n}^{\dagger}\left(a_{n}\right)$ creates (annihilates) an electronic excitation of energy $V_{n}$ at site $n$, and $J$ is the hopping integral. Any arbitrary Fibonacci system presents two kind of building blocks. In our case, we choose those blocks as individual centers $A$ and $B$, with $V_{n}=V_{A}$ or $V_{n}=V_{B} \cdot{ }^{1}$ The Fibonacci sequence $S_{n}$ is generated by appending the $n-2$ sequence to the $n-1$ one, i.e., $S_{n}=\left\{S_{n-1} S_{n-2}\right\}$. This construction algorithm requires the initial conditions $S_{0}=B$ and $S_{1}=A$. The $n$ sequence has $N=F_{n}$ elements, where $F_{n}$ denotes the $n$th Fibonacci number satisfying $F_{n}=F_{n-1}+F_{n-2}$ with $F_{0}=F_{1}=1$. As $n$ increases the ratio $F_{n-1} / F_{n}$ converges to $\tau=(\sqrt{5}-1) / 2=0.618 \ldots$, an irrational number which is known as the inverse golden mean. Therefore, lattice centers are arranged according to the Fibonacci sequence $A B A A B A B A \ldots$, where the fraction of $B$ centers is $c \sim 1-\tau$. 
The line shape $I(E)$ of an optical-absorption process in which a single exciton is created in a lattice with $N$ centers can be obtained as ${ }^{6}$

$$
I(E)=-\frac{2}{\pi N} \int_{0}^{\infty} d t e^{-\alpha t} \sin (E t) \operatorname{Im}\left(\sum_{n} G_{n}(t)\right),
$$

where the factor $\exp (-\alpha t)$ takes into account the broadening due to the instrumental resolution function of half width $\alpha$ and the correlation functions $G_{n}(t)$ obey the equation of motion

$$
i \frac{d}{d t} G_{n}(t)=\sum_{l} H_{n l} G_{l}(t),
$$

with the initial condition $G_{n}(0)=1$. The diagonal elements of the tridiagonal matrix $H_{n l}$ are $V_{n}$ whereas off-diagonal elements are simply given by $-J$.

\section{EXCITON-PHONON PROBLEM}

In the previous section we have summarized the lineshape problem at zero temperature. Now we deal with the exciton-phonon coupling and its influence on the opticalabsorption spectra. Under lattice vibrations the on-site energy will be subject to spatial and temporal fluctuations ${ }^{5}$ around their zero-temperature values, $V_{A}$ and $V_{B}$. By means of the Franck-Condon approximation and assuming that vibrational modes at different sites are independent, Schreiber and Toyozawa ${ }^{5}$ cast the problem into an equivalent one with random on-site correlations

$$
\left\langle V_{n} V_{n^{\prime}}\right\rangle=D^{2} \delta_{n n^{\prime}} .
$$

The approximations involved in writing Eq. (4) are correct in several cases of interest, such as optical modes in inorganic crystals. Therefore, we will consider in what follows the finite-temperature problem by using random on-site energies with Gaussian distribution

$$
P\left(V_{n}\right)=\left(\frac{1}{2 \pi D^{2}}\right)^{1 / 2} \exp \left(-\frac{\left(V_{n}-\langle V\rangle_{n}\right)^{2}}{2 D^{2}}\right),
$$

$\langle V\rangle_{n}$ being either $V_{A}$ or $V_{B}$ according to the Fibonacci sequence. Here $D^{2}=4 g J k_{B} T$ in one-dimension, where $g$ is the exciton-phonon coupling and $k_{B}$ is the Boltzmann's constant.

\section{NUMERICAL RESULTS AND DISCUSSIONS}

We have solved numerically the equation of motion (3) with random on-site energies distributed according to Eq. (5). As typical parameters we have chosen $V_{A}=4.0 \mathrm{eV}$, $V_{B}=4.8 \mathrm{eV}$, and $J=0.5 \mathrm{eV}$ so that the exciton bandwidth is $W=4 J=2.0 \mathrm{eV}$. In addition, we have taken $g=1$. We have checked that the main features of all lines of the spectra are independent of the system size. Hereafter we will take $N=F_{12}=233$ as a representative value. The width of the instrumental resolution was $\alpha=0.1 \mathrm{eV}$. Results at finite temperature comprise 25 thermal averages, i.e., averages over realizations of $V_{n}$.

In the pure $A$ lattice $\left(V_{n}=V_{A}\right)$ at zero temperature the spectrum consists of a single Lorentzian line centered at $E^{(A)}=V_{A}-2 J=3.0 \mathrm{eV}$. On increasing temperature this line

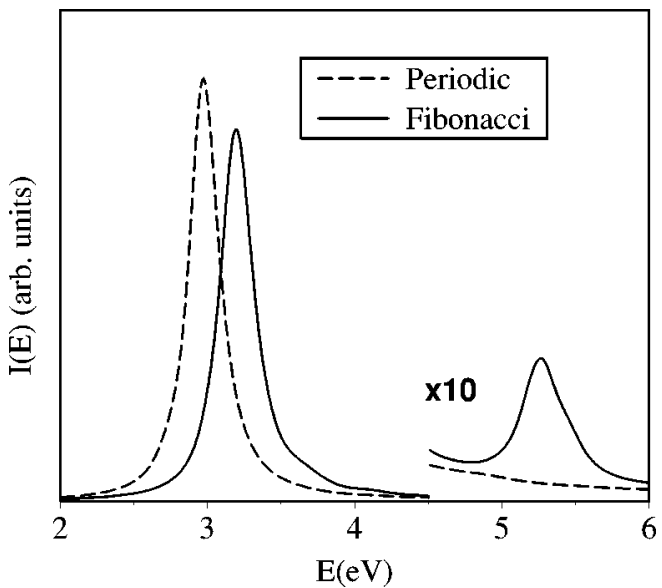

FIG. 1. Absorption spectra for a Fibonacci lattice (solid line) and a pure $A$ lattice (dashed line) at $T=77 \mathrm{~K}$.

is also Lorentzian-shaped for weak scattering $(D \ll W) .{ }^{5}$ Notice that the weak scattering case is an excellent approximation in the whole temperature range of interest. Figure 1 shows the results for a pure $A$ lattice at $T=77 \mathrm{~K}$. As soon as $B$ centers are introduced into the lattice according to the Fibonacci inflation rule, the main absorption line is shifted towards higher energies. The value of the energy shift can be computed using standard perturbation techniques to obtain $\Delta E=(1-\tau)\left(V_{B}-V_{A}\right) .{ }^{1}$ Therefore, the main absorption line of the Fibonacci lattice at zero temperature is centered at $E^{(A B)}=(1-\tau) V_{B}+\tau V_{A}-2 J=3.3 \mathrm{eV}$. Since we are in the weak scattering regime, the main absorption line is still Lorentzian, as it can be seen in Fig. 1 at $T=77 \mathrm{~K}$. Besides this main absorption line, a satellite line can also be observed in the high-energy part of the spectrum of the Fibonacci lattice, centered at about $5.2 \mathrm{eV}$ at zero temperature (see Fig. 1). Using again standard perturbation techniques, ${ }^{1}$ it is possible to demonstrate that a series of satellite peaks appear centered at

$$
E_{p}^{(A B)}=E^{(A B)}+4 J \sin ^{2}\left(\pi \tau^{p}\right),
$$

where $p=2,3, \ldots$. These satellite peaks are the fingerprint of the Davidov splitting ${ }^{7}$ since the Fibonacci lattice can be regarded as a crystal with a large unit cell. From Eq. (6) we obtain $E_{2}^{(A B)}=5.0 \mathrm{eV}$, close to the satellite observed in Fig. 1. Similarly $E_{3}^{(A B)}=4.2 \mathrm{eV}$ and so on. However, the remaining satellites are hidden by the main absorption line and cannot be observed in Fig. 1.

Figure 2 shows the absorption spectra of the Fibonacci lattice at different temperatures. We observe that the main absorption line is broadened and shifted to lower energies on increasing temperature. Most interestingly, the satellite line mentioned above is clearly observed even at room temperature. At higher temperature $(T=800 \mathrm{~K})$ the satellite is not well defined, as can be seen in Fig. 2. Some interesting physical conclusions can be drawn from these results. First of all, the quantum coherence required to observe the satellites in the high-energy region of the spectra is not completely lost even at room temperature. The satellite line is caused by the coupling of two excitonic modes, namely the lowest-lying and that with momentum $k=\tau^{2} N$, through the topology of the quasiperiodic lattice. ${ }^{1}$ At zero temperature 


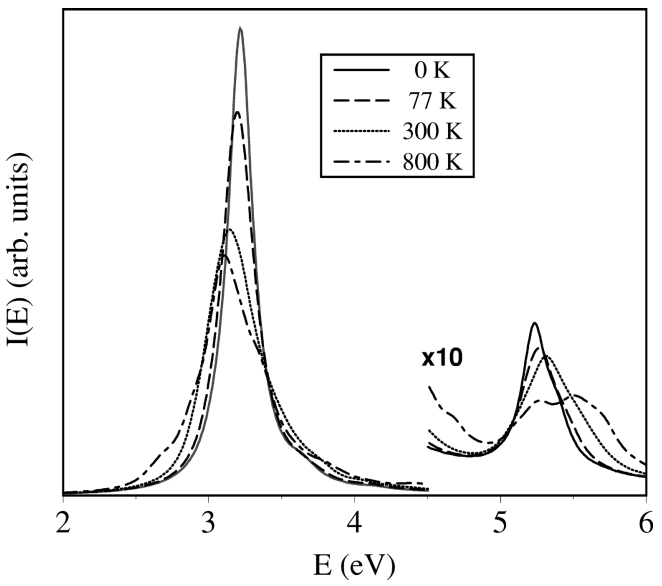

FIG. 2. Absorption spectra for a Fibonacci lattice at different temperatures: $T=0 \mathrm{~K}$ (solid line), $77 \mathrm{~K}$ (dashed line), $300 \mathrm{~K}$ (dotted line), and $800 \mathrm{~K}$ (dot-dashed line).

exciton wave functions extend over the whole lattice and see the long-range quasiperiodic order. On increasing temperature this coherence is partially lost but excitons still detect the long-range order. Further temperature increment (say, above room temperature with our model parameters) yields excitons so localized that they cannot see the quasiperiodic order. At this point it is useful to recall the concept of $\mathrm{co}^{-}$ herently bound centers. ${ }^{8,9}$ Due to finite-temperature effects, the number of coherently bound centers $N^{*}$ is smaller than the system size $N$. This parameter can be determined from ${ }^{8,9}$

$$
N^{*}=\left(3 \pi^{2} \frac{J}{D}\right)^{2 / 3}=\left(\frac{9 \pi^{4} J}{4 g k_{B} T}\right)^{1 / 3} .
$$

${ }^{1}$ F. Domínguez-Adame, Phys. Lett. A 217, 59 (1996).

${ }^{2}$ E. Maciá and F. Domínguez-Adame, Phys. Rev. B 50, 16856 (1994).

${ }^{3}$ F. Domínguez-Adame, E. Maciá, and A. Sánchez, Phys. Rev. B 51, 878 (1995).

${ }^{4}$ F. Domínguez-Adame and E. Maciá, Phys. Rev. B 53, 13921 (1996).
Since at room temperature the characteristic features of the long-range order are still present in the optical-absorption spectra, whereas at $T=800 \mathrm{~K}$ they cannot be detected, we can conclude there exists a critical value of the number of coherently coupled centers to probe the long-range order. We can estimate this critical number from Eq. (7) to lie between $N^{*}(T=300 \mathrm{~K})=16$ and $N^{*}(T=800 \mathrm{~K})=12$.

\section{CONCLUSIONS}

In summary, we have studied numerically the absorption spectra of Frenkel excitons on Fibonacci lattices. Excitonphonon coupling has been taken into account because it is a major limiting factor of the quantum coherence. This coherence is responsible for satellite lines in the high-energy region of the optical spectrum. We have demonstrated that the characteristic features due to the long-range order of Fibonacci lattices are observed even at room temperature. The number of coherently bound centers needed to probe the long-range order turns out to be of the order of $N^{*}=15$. Thus, excitons are indeed a probe of the underlying quasiperiodicity even at room temperature. This conclusion should facilitate future experimental work on linear systems with long-range order.

\section{ACKNOWLEDGMENTS}

The authors thank A. R. Bishop, E. Maciá, V. Malyshev, and A. Sánchez for helpful comments and discussions. This work is supported by CICYT through Project No. MAT950325 .
${ }^{5}$ M. Schreiber and Y. Toyozawa, J. Phys. Soc. Jpn. 51, 1528 (1982).

${ }^{6}$ D. L. Huber and W. Y. Ching, Phys. Rev. B 39, 8652 (1989).

${ }^{7}$ A. S. Davidov, Theory of Molecular Excitons (Plenum, New York, 1971).

${ }^{8}$ V. A. Malyshev, Opt. Spectrosc. 71, 505 (1991).

${ }^{9}$ V. A. Malyshev and P. Moreno, Phys. Rev. B 51, 14587 (1995). 